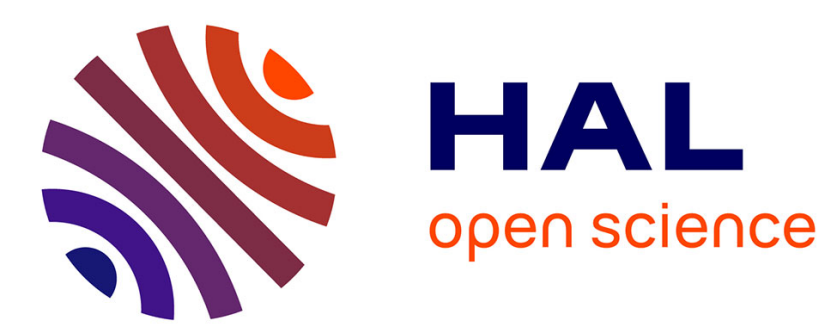

\title{
The Shear Modulus of Liquids
}

A. Granato

\section{To cite this version:}

A. Granato. The Shear Modulus of Liquids. Journal de Physique IV Proceedings, 1996, 06 (C8), pp.C8-1-C8-9. 10.1051/.jp4:1996801 . jpa-00254518

\section{HAL Id: jpa-00254518 https://hal.science/jpa-00254518}

Submitted on 1 Jan 1996

HAL is a multi-disciplinary open access archive for the deposit and dissemination of scientific research documents, whether they are published or not. The documents may come from teaching and research institutions in France or abroad, or from public or private research centers.
L'archive ouverte pluridisciplinaire HAL, est destinée au dépôt et à la diffusion de documents scientifiques de niveau recherche, publiés ou non, émanant des établissements d'enseignement et de recherche français ou étrangers, des laboratoires publics ou privés. 


\title{
The Shear Modulus of Liquids
}

\author{
A.V. Granato \\ University of Illinois, Department of Physics, 1110 West Green Street, Urbana, IL 61801, U.S.A.
}

\begin{abstract}
According to the interstitialcy model of condensed matter states, liquids are crystals containing a few percent of self-interstitials in thermal equilibrium. Glasses are frozen liquids. The model is expressed in terms of the properties of the shear modulus, which decreases exponentially with the interstitial concentration and temperature in the liquid state, and can be nearly as large as the crystalline value in the supercooled liquid near the glass temperature. Available experimental data support the model predictions, showing that the shear modulus plays a central role in the description of thermodynamic and kinetic properties of liquids and glasses.
\end{abstract}

\section{INTRODUCTION}

What does the shear modulus of liquids have to do with anelasticity in solids? Firstly, glasses are solids whose structure is that of frozen liquids. Secondly, according to the interstitialcy model of condensed matter states [1] liquids and glasses are crystals containing a few percent of interstitials. Thirdly, the shear modulus plays a central role in this model.

Of the various physical properties of matter, we focus here on the shear modulus because of certain advantages of interpretation and measurement it provides. Results for the shear modulus, while non-intuitive and surprising, are simple and clear. Also the shear modulus is one of the more accurately measurable properties available. In addition, as already mentioned, the shear modulus play a key role in the interstitialcy model of condensed matter states.

It is widely believed that the shear modulus of a liquid is zero. In fact, this is often taken as a definition of the liquid state and a number of theories of melting are based on this assumption. But it is not true. The interstitialcy model predicts that the shear modulus of a liquid can be nearly as large in the supercooled equilibrium state as that for the crystalline state. A few measurements on amorphous materials near the glass temperature are available which show reversible (equilibrium) effects which can be readily understood with this model in terms of the liquid state shear modulus, but which are otherwise incomprehensible.

An analytic expression for the Gibbs free energy as a function of temperature, pressure, shear stress and concentration has been derived, from which all thermodynamic properties of the material in each of the crystalline, liquid or amorphous states, as well as their changes on melting, can be obtained. The tunneling entity providing the two-level system (TLS) behavior found for low temperature properties was identified as the interstitialcy configuration. The model gives a sound physical basis for the Lindeman relation for melting, and provides many testable propositions.

In the next section, we first review briefly the salient features of the model, and in the third section, we discuss a sample of evidence concerning the temperature dependence of the shear modulus in each of the crystalline, liquid and glassy states. In Section IV, the temperature dependence of the shear modulus is used to discuss other thermodynamic and kinetic properties. 


\section{THE INTERSTITIALCY MODEL}

Liquids and amorphous materials must have structures and dynamics consisting of motions about static equilibrium configurations which are mechanically stable and thermodynamically metastable below the melting temperature. The types of intrinsic defects a crystal can have are very limited and known: vacancies and interstitials as point defects, dislocations and disclinations as line defects, surfaces, stacking faults and twins as planar defects, or combinations of these. Linear and planar defects have energies which are prohibitively high for generation by thermal activation.

In pioneering calculations by Huntington and Seitz [2] made for copper, it was found that the interstitial has a higher formation and a lower migration energy than does a vacancy, but it was not possible to determine the stable interstitial equilibrium configuration. Early estimates of point defect contributions expected for the shear modulus, volume and entropy of point defects are given in Table I. Here $c$ is the defect concentration, $s$ the entropy per defect in Boltzmann units, $V$ is the volume and $\Omega$ is the atomic volume.

Table I: Estimates of Defect Contributions Expected for Some Thermodynamic Properties of Copper.

\begin{tabular}{|c|c|c|c|}
\hline \multirow[t]{2}{*}{ Property } & \multirow[t]{2}{*}{ Vacancies } & \multicolumn{2}{|c|}{ Interstitials } \\
\hline & & Octahedral & $<100>$ dumbbell \\
\hline$\frac{1}{G} \frac{d G}{d c}$ & ${ }^{-1},(b)$ & $\begin{array}{c}+5 \\
(\mathrm{a}),(\mathrm{b})\end{array}$ & $\begin{array}{l}-20 \\
(\mathrm{~g})\end{array}$ \\
\hline$\frac{1}{\mathrm{k}} \frac{\mathrm{ds}}{\mathrm{dc}}$ & (c), (d), (e) & $\begin{array}{c}0.8 \\
\text { (c), (d), (e) }\end{array}$ & $\begin{array}{l}10-20 \\
\text { (h) }\end{array}$ \\
\hline$\frac{1}{\Omega} \frac{d V}{d c}$ (f) & 0.7 & 0.7 & 0.7 \\
\hline$\frac{\Omega}{\mathrm{k}} \frac{\mathrm{ds}}{\mathrm{dV}}$ & 2.1 & 1.1 & $14-28$ \\
\hline
\end{tabular}

(a) Dienes, Ref. 3

(b) Nabarro, Ref. 4

(c) Huntington, et. al., Ref. 5

(d) Dienes, Ref. 6 (e) Zener, Ref. 7

(f) Summary in Erhart, Ref. 8

(g) Dederichs, et. al., Ref. 9

(h) see text

It is difficult to distinguish between defects with volume measurements, but the shear modulus is expected to be selective. There were, and still are, no direct measurements of interstitial entropies.

Early measurements of shear modulus changes with Frenkel pairs in pure copper produced by low temperature irradiation did indeed show increases, but often one or two orders of magnitude larger than expected. It was soon realized that this was the result of dislocation pinning. When dislocation pinning is suppressed, measurements of the change of the three independent elastic constants of copper as a function of Frenkel pair concentration produced by neutron irradiation at helium temperature [10] are as shown in Fig. 1.

The elastic constants decrease linearly with defect concentration, $c$. For the annealing range $I_{D}$ where interstitials disappear, the changes are found to be $d\left(\ln C_{i j}\right) / d c=-31,-15$, and 0 for $C_{44}, C^{\prime}=$ $\left(C_{11}-C_{12}\right) / 2$, and $B$, respectively, where $B$ is the bulk modulus. The change in $C_{44}$ is very large. Changes of most properties, such as the volume or bulk modulus, are of order unity. The change of $\mathrm{C}_{44}$, if extrapolated linearly, would imply that the lattice becomes unstable for about $3 \%$ of interstitials. The 


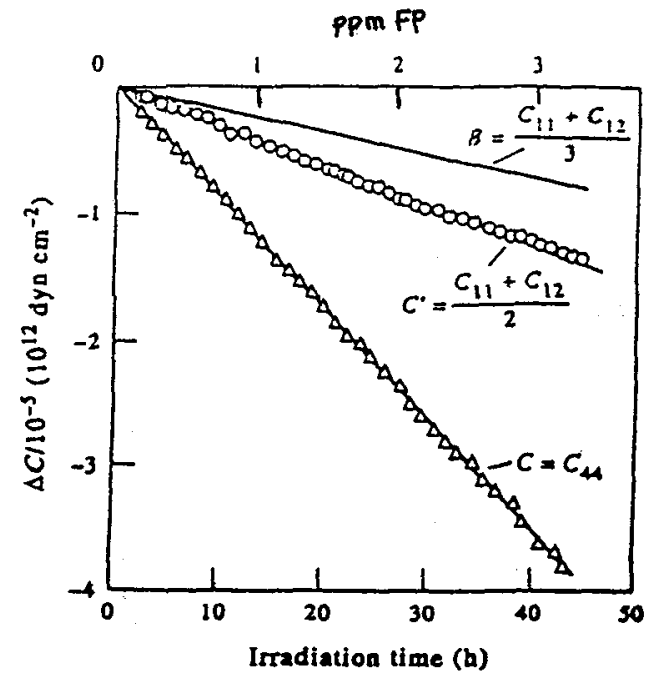

Figure 1: Elastic constant changes in copper as a function of Frenkel pair concentration. (a)
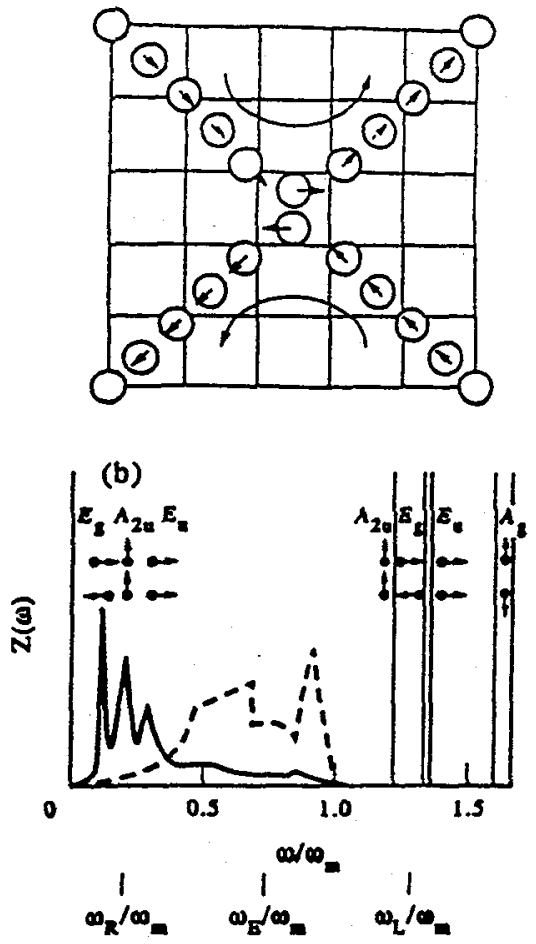

Figure 2(a): dumb-bell interstitiaicy configuration in fee metals. Arrows indicate displacements of atoms along close-packed $\langle 110\rangle$ directions for an applied shear stress and the $\mathrm{Eg}$ resonance mode. (b) Calculated lattice phonon spectrum (--) resonance, $\omega \mathrm{g}$, and local modes, $\omega_{\}}$, for copper [9]. The symmetry and displacements of the central dumb-bell atoms are indicated for the resonance and local modes. For a high-temperature approximate calculation, we replace the lattice frequencies by the single Einstein frequency $\omega_{\mathrm{E}}$, the resonance modes by a single $\omega_{R}$, and the local modes by a single $\omega_{L}$, as indicated at the bottom of the figure.

magnitude and anisotropy of the shear constant changes are strong evidence $[9,10]$ for the dumb-bell interstitialcy configuration of the interstitial, shown in Fig. 2(a).

The dumb-bell displacements are aligned in $\langle 100\rangle$ directions and are strongly coupled to atoms in the close-packed $<110\rangle$ directions, so that the configuration consists of a $<100\rangle$ dumb-bell extended with appreciable chains for several atoms along $\langle 110\rangle$ directions. The stable and saddle-point configurations are reversed for an interstitial atom from that for a vacancy. This difference of symmetry has far-reaching consequences. In particular, the interstitialcy configuration has a strong coupling to external shear stress, low-frequency resonance modes [9], and an extended linear string-like character which can lead to large entropy effects. It has been pointed out by Schober [11] that many features of low-temperature glass anomalies (low-frequency vibrations, relaxation processes, and general TLS behavior) are also observed in crystals after irradiation at doses much less than those needed for amorphization. The vibrational frequency spectrum of fcc crystals containing isolated interstitialcies has been described in detail by Dederichs et al. [9]. They find both low-frequency resonance modes and 
high-frequency local modes as shown in Fig. 2(b). The results depend somewhat on the interatomic potential chosen.

Three features, (1) a degeneracy in orientation, (2) a strong softening of the shear modulus and (3) resonance modes, which lead to a large entropy for interstitials, are the essential ingredients of a quantitative model for condensed matter states of simple materials.

A simple estimate of the entropy change per defect on melting is given by $\Delta s=5 \mathrm{kln} \omega_{\mathrm{E}} / \omega_{\mathrm{R}} \sim 10 \mathrm{k}$. This should be a lower limit since the chain of atoms closely coupled along $<110\rangle$ directions is stringlike and can have overtones. A self-consistency argument using the definition of the shear modulus $G \equiv$ $\left(\delta^{2} \mathrm{~F} / \delta \varepsilon^{2}\right) \mathrm{N} \Omega_{0}$, recognizing that the shear modulus must be periodic in displacements which repeat the lattice structure, leads to

$$
\mathrm{G}=\mathrm{G}(\mathrm{V}, \varepsilon) \exp (-\beta c),
$$

for the concentration dependence of the shear modulus, where $\beta$ is the diaelastic shear susceptibility.

The result, eqn (1), is basic for the model. It describes a large diaelastic softening of the shear modulus and of the free energy with defect concentration. With $\beta \sim 20-30$, a concentration of a few percent of interstitialcies should greatly reduce the shear modulus, in agreement with the results shown in Fig. 1.

The changes at the melting transition are $\Delta V / \Omega \sim 0.045$ and $\Delta S / k \sim 1.15$. For a defect model of melting we should then expect $(\Delta S / k) /(\Delta V / \Omega)$ to be $\sim 26$ for the melting transition, but the expected value of this ratio for copper from Table 1 is 2.1 for vacancies, and 1.1 for octahedral symmetry interstitials. The discrepancy of an order of magnitude is large enough to rule these out. Theories have been attempted for Frenkel pairs, for which atoms are supposed to jump thermally from lattice sites to interstitial sites. This would lead to homogeneous melting, contrary to the heterogeneous melting known from experiment. Since these are the only defects known from solid-state physics, we should then expect dumbbell interstitials (the interstitialcy configuration) to be the source.

The equilibrium interstitial concentration increases exponentially with temperature in the crystalline state, as with vacancies, but remains small even at the melting temperature. In contrast, the equilibrium concentration in the liquid state is relatively large, but increases approximately linearly with temperature. 'Then, expressing $\Delta \mathrm{c}=(\mathrm{dc} / \mathrm{dT})_{\mathrm{g}}(\mathrm{T}-\mathrm{Tg})$ in eqn. 1 , we have

$$
\mathrm{G}^{\mathrm{l}}=\mathrm{G}_{\mathrm{g}} \exp (1-\mathrm{T} / \mathrm{Tg}), \quad \mathrm{T}>\mathrm{Tg}
$$

where $\mathrm{Gl}^{\mathrm{l}}$ is the liquid-state shear modulus, $\mathrm{Gg}$ is the shear modulus at the glass temperature $\mathrm{T}_{\mathrm{g}}$, and $\gamma=\beta \mathrm{Tg}(\mathrm{dc} / \mathrm{dT}) \mathrm{g}$. For $\gamma \sim 3$, a $25 \%$ increase of $\mathrm{T}$ above the glass temperature should decrease $\mathrm{G}^{\mathrm{l}}$ by about a factor of two. The expected temperature dependence of the shear modulus in each of the crystalline $\left(G_{X}\right)$, liquid $\left(G^{l}\right)$, and glassy or amorphous $\left(G_{g}\right)$ states is then as shown in Fig. 3.

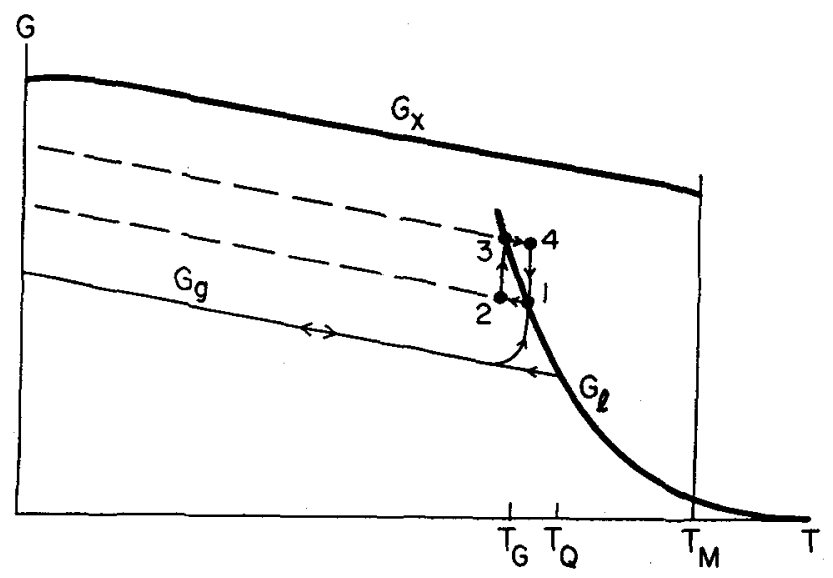

Figure 3. Schematic sketch of the shear modulus as a function of temperature in the crystalline $\left(G_{x}\right)$, liquid $\left(G_{1}\right)$, and glassy $\left(G_{g}\right)$ states. $T M$, $T_{Q}$ and $T_{G}$ are the melting, freezing temperature for a fast quench and freezing temperature for a slow quench. respectively. Points $1,2,3,4$ are states of a reversible cycle of measurement. The bold lines are for the crystalline and liquid equilibrium states. The light lines are for non-equilibrium glassy states. 
In the figure, $T_{M}$ is the melting temperature, $T_{Q}$ is the freezing-in temperature for a fast quench, and $T_{G}$ is the freezing-in temperature for a slow quench.

The shear modulus is small, but not zero in the liquid state above the melting temperature. It is normally not detected above the melting temperature because the elastic response of the material is overwhelmed by the viscous flow at these temperatures where the viscosity $\eta$ is low. The thermodynamic equilibrium liquid-state shear modulus $G^{l}(T)$ is given by the high frequency $\omega$ limit $\omega T \gg 1\left(G_{\infty}\right)$ for $G(\omega)$, with the time constant $\tau$ for a small temperature change given by the Maxwell relation $\tau=\eta / G$.

The shear modulus $\mathrm{G}^{\mathbf{l}}$ increases rapidly as the temperature decreases below the melting temperature in the equilibrium supercooled liquid state. For a rapid quench, the liquid freezes at $T_{Q}$ and then has the same temperature dependence for lower temperatures as in the equilibrium crystalline state $\left(G_{x}\right)$. If the temperature is then increased at a lower rate, the modulus increases toward the equilibrium liquid state value near $\mathrm{T}_{\mathrm{G}}$. If now, the temperature is decreased a small amount quickly, the modulus increases instantaneously (1-2), and then further more slowly (2-3), until the equilibrium liquid value (3) is achieved. If now the temperature is increased quickly back to the starting value (3-4), the modulus decreases instantaneously, and then more slowly back to the liquid equilibrium value (4). The change (41 ) is however much more rapid than that for (2-3) because the viscosity is smaller at the higher temperature. This cycle (1-2-3-4-1) is reversible and may now be repeated because points 1 and 3 are equilibrium states.

\section{EXPERIMENTAL RESULTS}

\subsection{Acoustic delay times in three metallic glasses.}

Measurements by Chen [12] of acoustic delay times of three metallic glasses as a function of temperature are shown in Fig. 4. The transit time is determined by Young's modulus. Young's modulus $\mathrm{E}$ is closely related to the shear modulus by $E=3 G /[1+G / 3 B]$ so that one would then expect the Young's modulus changes also to be large. When the specimens are held well below the glass temperature $T_{\mathrm{g}}$, the measurements are

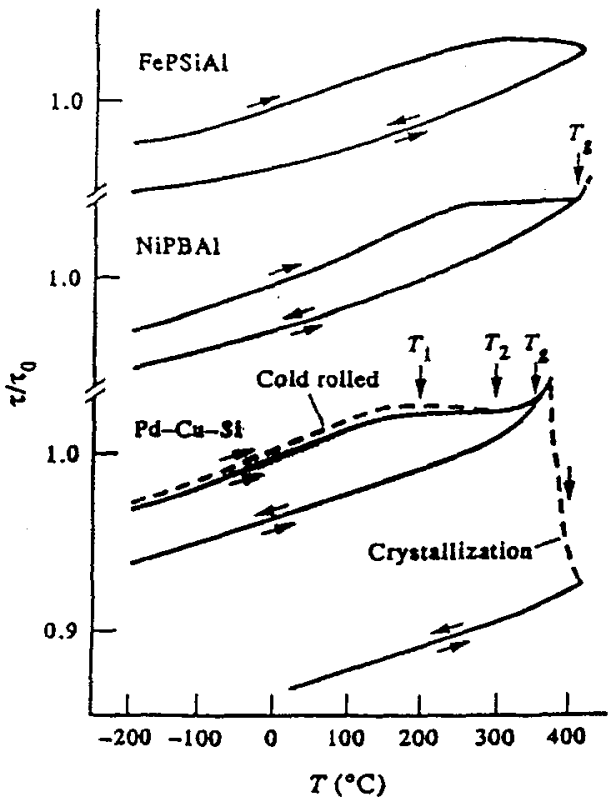
reversible because the defects are frozen in. When the specimens are brought near $T_{g}$, Young's modulus increases. Above $T_{\mathrm{g}}$, the specimen crystallizes. Just before crystallization, the transit time increases as the specimen $\mathrm{Pd}-\mathrm{Cu}-\mathrm{Si}$ enters the supercooled liquid region. It thus appears that these measurements are the earliest to show the features. predicted in Fig. 3 for all three of the crystalline, glassy, and supercooled liquid states. The supercooled region is very limited, however.

Figure 4: Reduced delay time $\tau / \tau 0$ metallic glasses, $\mathrm{Pd}_{77.5} \mathrm{Cu}_{6} \mathrm{Si}_{16.5}, \mathrm{Ni}_{75} \mathrm{P}_{16} \mathrm{~B}_{6} \mathrm{Al}_{3}$, and $\mathrm{Fe}_{75} \mathrm{P}_{16} \mathrm{Si}_{6} \mathrm{Al}_{3}$, where $\tau_{0}$ is the delay time of the initial glass sample, $T_{g}$ is the glass transition temperature, and $T_{1}$ and $T_{2}$ are the temperatures at which the rates of irreversible change in $\tau$ are maxima. 


\section{$3.2 G^{1}(T)$ and $G \mathrm{~g}(T)$ as a function of annealing near $T_{\mathrm{g}}$.}

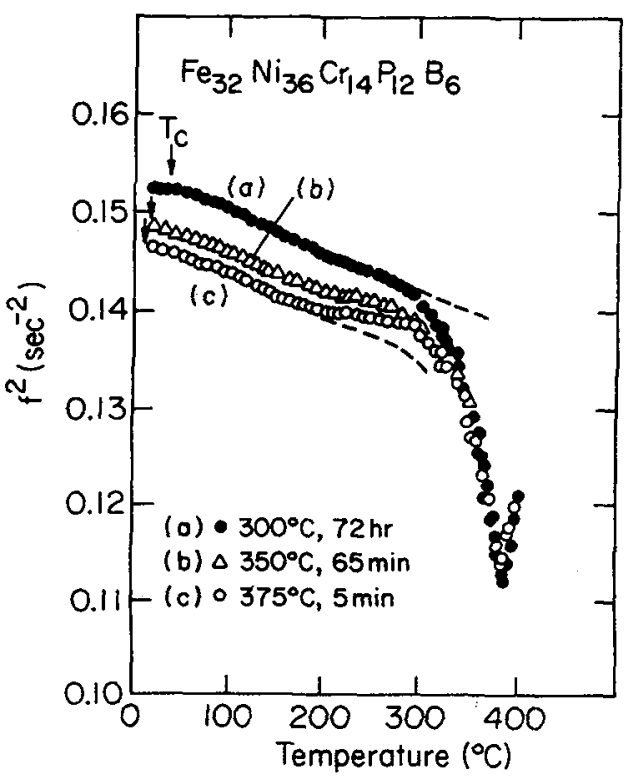

Torsion pendulum data for $\mathrm{G}(T)$ for $\mathrm{Fe}_{32} \mathrm{Ni}_{36} \mathrm{Cr}_{14} \mathrm{P}_{12} \mathrm{~B}_{6}$ by Morito and Egami [13] are shown in Fig. 5 for samples brought to quasi-equilibrium at three different temperatures. In the glassy state below $300^{\circ} \mathrm{C}$ the magnitude of the shear modulus depends on the annealing temperature, but does not in the supercooled region above $350^{\circ} \mathrm{C}$. The temperature dependence in the supercooled liquid region is much greater than in the glassy region. At $385^{\circ} \mathrm{C}$, the samples recrystallize. A small change near $0^{\circ} \mathrm{C}$ is due to a magnetic transition at $T_{c}$, shown by arrows.

Figure 5: Temperature dependence of $\mathrm{f}^{2}$, therefore $G$, for samples preannealed and brought to quasiequilibrium at three different temperatures. The sharp increase above $385^{\circ} \mathrm{C}$ is due to crystallization. (After Morito and Egami[13]).

\subsection{Supercooled liquid state shear compliance in molecular liquids.}

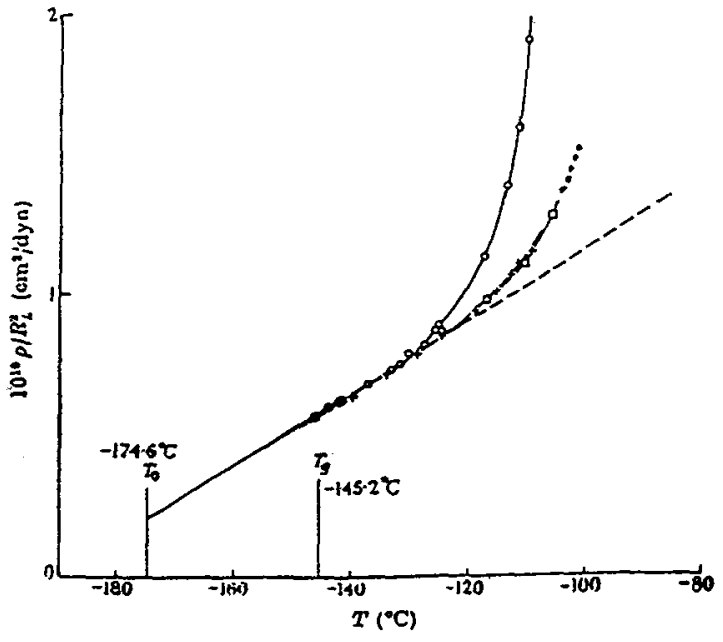

Figure 6: Measured values of $\rho / R_{L}^{2}$ against temperature for sec. -butyl benzene showing the linear variation of $1 / \mathrm{G}_{\infty}$ with temperature $0,30 \mathrm{MC} / \mathrm{s} ;+, 450 \mathrm{Mc} / \mathrm{s} ; 0,1000 \mathrm{Mc} / \mathrm{s}$. (After Barlow, et: al. [14])
The shear wave properties of a number of molecular liquids as a function of temperature for several frequencies were determined by Barlow, et al. from measurements of the complex reflection coefficient of an incident shear wave at a solid/liquid interface. Fig 6 shows the temperature dependence of the shear compliance $J(\omega)=1 / G(\omega)$ for sec -butyl benzene for about $40^{\circ} \mathrm{C}$ above the glass temperature of $-145.2^{\circ} \mathrm{C}$ at three frequencies. At the lowest temperatures, the curves agree for all frequencies and give $J_{\infty}=1 / G_{\infty}(T)=1 / G^{1}(T)$. The authors fit this data to a linear temperature dependence (dashed line), and suppose the pulling away from the line results from viscoelastic behavior at high temperatures. However, an exponential temperature dependence, as predicted by Eq. 2 and fitted to the lowest temperature data, is shown as a dotted line and fits the data for 450 and $1000 \mathrm{MHz}$. The fact that the data are the same for the higher frequencies shows that this is the equilibrium curve $1 / G^{\mathrm{l}}(T)$, and the fit shows that the temperature dependence of 


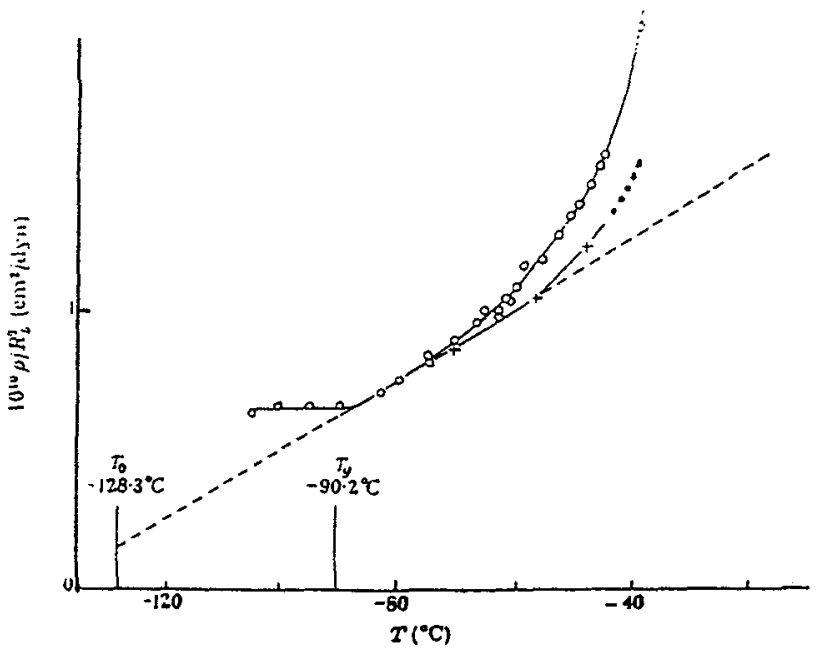

$\mathrm{G}^{\mathrm{l}}(\mathrm{T})$ is exponential, as predicted by eqn 2.

Similar data for di(isobutyl) phthalate are shown in Fig. 7, but these are carried to temperatures below $\mathrm{T}_{\mathrm{g}}$. An abrupt change to a lower temperature dependence is seen at $T_{g}$.

Figure 7: Measured values of $\rho / R_{L}^{2}$ against temperature for di(isobutyl) phthalate showing the linear variation of $1 / G_{\infty}$ with temperature. Points at temperatures below $\mathrm{T}_{\mathrm{g}}$ are for the nonequilibrium glass, $0,30 \mathrm{MC} / \mathrm{s} ;+, 450 \mathrm{Mc} / \mathrm{s}$; . (After Barlow, et. al. [14])

\subsection{Young's Modulus in the crystalline, glassy and supercooled liquid states for three} bulk metallic glasses.

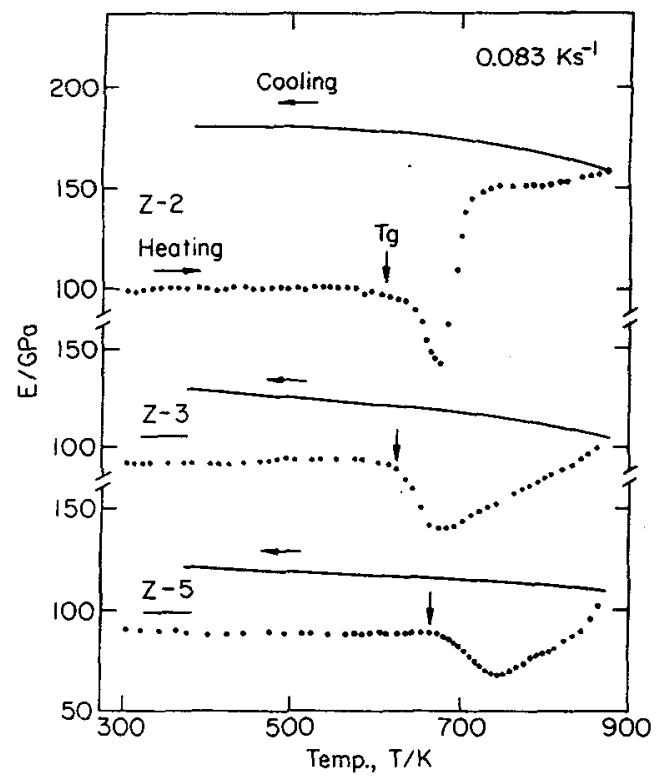

Kimura, et al.[15] have measured Young's modulus in bulk metallic glasses with a wide supercooled liquid region, providing results for the crystalline, glassy and supercooled liquid states in the same material. Young's modulus was measured by an electrostatic driving method at frequencies $300 \sim 600 \mathrm{~Hz}$ at temperatures from 300 to $900 \mathrm{~K}$. Results for 3 compositions are shown in Fig. 8. Above the glass temperature, the Young's Modulus decreases with a temperature dependence much greater than the glass or the crystal until recrystallization occurs. The magnitude of the changes depends some-what on the system, but is qualitatively similar for all. The ratios of the shear modulus in the glassy state to those in the crystalline state lie in the range of $55-75 \%$.

Figure 8: Temperature dependence of Young's modulus $(E)$ of $\mathrm{Zr}_{67} \mathrm{Cu}_{33}(\mathrm{Z}-2), \mathrm{Zr}_{65} \mathrm{Al}_{7.5} \mathrm{Cu}_{27.5}(\mathrm{Z}-3)$ and $\mathrm{Zr}_{60} \mathrm{Al}_{15} \mathrm{Cu}_{2.5} \mathrm{Ni}_{7.5} \mathrm{Cu}_{15}(\mathrm{Z}-5)$. (After Kimura, et. al. [15]).

\subsection{Cyclic reversible behavior of Young's Modulus in an FeNiMoB amorphous alloy.}

Allia, et al. [16] have measured Young's Modulus in $\mathrm{Fe}_{40} \mathrm{Ni}_{38} \mathrm{Mo}_{4} \mathrm{~B}_{18}$ obtained at different quenching rates, using the decay of electrostatically excited flexure vibrations in the $10^{2}-10^{3} \mathrm{~Hz}$ range. Reversible variations of the modulus are observed (Fig. 9) by cycling between two different temperatures after a long aging time $\left(10^{4}-10^{5} \mathrm{sec}\right)$ at the higher one. 


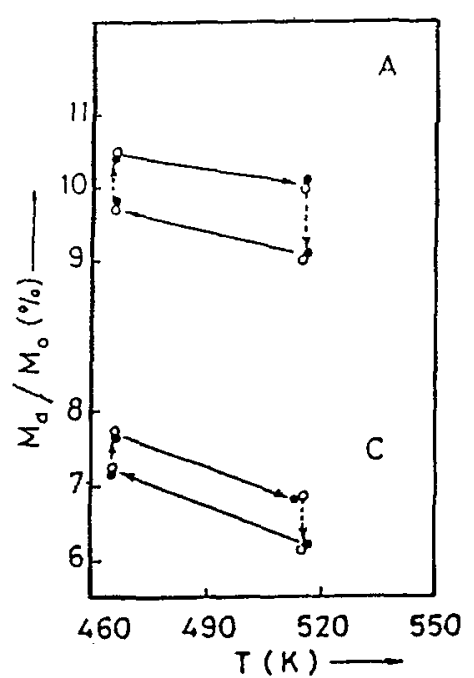

Figure 9: Cycling effect between 495 and $515 \mathrm{~K}$ on $M_{d}$ for series $A$ and $C$ specimens after $10^{4} \mathrm{sec}$ ageing at $515 \mathrm{~K}$. Dashed arrows isothermal treatments ( 40 mins); full arrows fast $T$ changes. Open circles first run, full circles second run. (After Allia, et al. [16])

\subsection{Summary of measurements.}

All of the effects predicted in Fig. 3 with Eq. 2 are found in these measurements. The magnitudes and temperature dependences are in reasonable quantitative agreement with the expectations of the interstitialcy model, providing further support for the model.

\section{OTHER THERMODYNAMIC AND KINETIC EFFECTS IN SUPERCOOLED LIQUIDS}

It's been shown previously [17] that the liquid state specific heat should be larger than the crystalline specific heat by an amount which is proportional to the interstitial formation energy. This in turn is proportional to the shear modulus which decreases exponentially with temperature. A more mysterious and characteristic property of glass-forming materials is the non-Arrhenius temperature dependence of the viscosity. A satisfactory explanation of this temperature dependence has been the goal of many theoretical efforts over many years. Recently, a fascinating proposal has been made by Dyre, et al. [18] which has promise for a solution to the non-Arrhenius temperature dependence puzzle. They express the viscosity by

$$
\eta=\eta_{0} \exp (\mathrm{F} / \mathrm{kT})
$$

where $\eta_{0}$ is a reference viscosity, and they suppose that $\mathrm{F}$ is given by the work required to shove aside neighboring particles for a particle in a diffusing process. To estimate $F$, they use the familiar sphere-inhole elasticity problem, and obtain

$$
\mathrm{F}=\mathrm{GV}_{\mathrm{c}}
$$

where $V_{c}$ is a characteristic volume. But $G$ is the shear constant of the liquid in the supercooled regime which is not given theoretically. By using the known measured temperature dependence of the infinite frequency limit $G_{\infty}(T)$ for about a dozen molecular liquids, they find reasonable agreement with the measured non-Arrhenius temperature dependence of the viscosity.

With the interstitialcy model, the liquid state shear modulus is given, so that eqn. 2 can be used to describe and analyse $\eta(T)$. 


\section{SUMMARY}

The interstitialcy model predicts that the liquid state shear modulus decreases exponentially with temperature, as described by Fig. 3 and eqn. 2. Available data support this prediction providing further evidence for the model and the central role played by the shear modulus. Its absolute value and temperature dependence can be measured to a far higher accuracy than can be given by ab-initio theories. Other thermodynamic and kinetic properties are also expressible in terms of the shear modulus. The selfinterstitial, which plays a secondary role to the vacancy in the crystalline state, except for radiation effects, becomes the basic structural unit for liquids and glasses. The shear modulus is also elevated in status, becoming a convenient and practical instrument for describing properties of liquid and glassy states.

\section{ACKNOWLEDGMENT}

This work was supported by the National Science Foundation under grant DMR93-19773.

\section{REFERENCES}

[1] Granato A.V., Phys. Rev. Lett. 68 (1992) 974.

[2] Huntington H. B., and Seitz F., Phys. Rev. 61, (1942) 315; Huntington H. B., Phys. Rev. 91 (1953) 1092.

[3] Dienes, G. J., Phys. Rev. 86, (1952) 228; 87 (1952) 666.

[4] Nabarro, F. R. N., Phys. Rev. 87 (1952) 665.

[5] Huntington, H. B., Shirn, G. A., and Wajda, E. S., Phys. Rev. 99 (1955) 1085.

[6] Dienes, G. J., Phys. Rev. 89 (1953) 185.

[7] Zener, C. J, Appl. Phys. 22 (1951) 372

[8] Ehrhart, P. Landolt-Börnstein, New Series III/25, Springer Verlag (1991) 88.

[9] Dederichs P. H., Lehmann C., Schober H. R., Scholz A., and Zeller R., J. Nucl. Mater. 69, (1978) 176.

[10] Holder J. T., Granato A. V., and Rehn L. E., Phys. Rev. Lett. 32 (1974) 1054; Phys. Rev. B10 (1974) 363; Rehn L. E., Holder J. T., Granato A. V., Coltman R. R. and Young F. W., Jr., Phys. Rev. 10 (1974) 349.

[11] Schober H. R., Phonon 89 (Edited by S. Hunklinger, W. Ludwig and G. Weiss) Vol. 1, p. 444. World Scientific, Singapore (1989).

[12] Chen H. S., J. Appl. Phys. 49 (1978) 3289.

[13] Morito N., and Egami, T., J. NonCryst. Solids 61\&62 (1984) 973.

[14] Barlow A. J., Lamb J., Matheson A. J., Padmini P. R. K. L., and Richter J., Proc. R. Soc. London Ser. A 298 (1967) 467.

[15] Kimura H., Kishida M., Kaneko T., Inoue A., and Masumoto T. Materials Transactions, JIM, 36 (1995) 890.

[16] Allia P., Bonetti E. Evangelista E., and Vinai, F., J. de Physique C10, 46 (1985) 481

[17] Granato A. V., J. Phys. Chem. Sol. 55 (1994) 931.

[18] Dyre J. E., Olsen, N. B. and Christensen, T., Phys. Rev B 53 (1996) 2171. 